\title{
Can Clinicians Start Prescribing Inclisiran for Hypercholesterolemia Today? A Review of Clinical Studies for Internal Medicine Physicians and Endocrinologists
}

\author{
Abdullah Jahangir $^{1}$, Syeda Sahra ${ }^{1}$, Michael Krzyzak ${ }^{1}$ \\ 1. Internal Medicine, Northwell Health, Staten Island, USA
}

Corresponding author: Abdullah Jahangir, ajahangir@northwell.edu

\begin{abstract}
The safety profile and efficacy margin of inclisiran as a lipid-lowering drug have been assessed in clinical trials and are underway in subgroups with relevant co-morbidities. This systematic review looks at the clinical trials that have been conducted to comment on its safety and efficacy. The conclusions can serve as a guide for practicing physicians and researchers for following current and future cohorts of patients. PubMed, Cochrane, Embase, Scopus, CINAHL, Web of Science, and Clinicaltrials.gov were searched comprehensively using the terms "Inclisiran", "ALN-PCSsc", and "ALN-PCS” using the Boolean operator "OR" with data cut-off date of June 28,2020 . The outcomes of safety and efficacy were collected and charted for the systematic review. In our study, eight clinical trials were included in the final study: the ORION $(1,2,7,9-11)$ trials and two clinical trials (phase 1 randomized clinical trials) done before ORION trials. Favourable efficacy in terms of LDL levels and PSCK9 levels was observed across all eight clinical trials. No severe adverse effects, safety concerns, or fatalities attributable directly to inclisiran were reported. Therefore, our study results suggest a positive efficacy and safety profile of inclisiran as a lipid-lowering drug in clinical trials.
\end{abstract}

Review began 07/04/2021 Review ended 07/07/2021 Published 07/27/2021

\section{() Copyright 2021}

Jahangir et al. This is an open access article distributed under the terms of the Creative Commons Attribution License CC-BY 4.0., which permits unrestricted use, distribution, and reproduction in any medium, provided the original author and source are credited.
Categories: Endocrinology/Diabetes/Metabolism, Family/General Practice, Internal Medicine Keywords: inclisiran, pcsk9 inhibitors, aln-pcs

\section{Introduction And Background}

Proprotein convertase subtilisin/kexin type 9 (PCSK9) is encoded by the PCSK9 gene, and it regulates the hepatic low-density lipoprotein (LDL) receptors. It is secreted in the liver and modulates the activity of LDL receptors. PCSK9 binds the LDL receptors in their active form, leading to their lysosomal destruction [1, 2]. Once PCSK9 levels were linked with hypercholesterolemia, there have been many trials to assess different agents which block this interaction between PCSK9 and LDL receptors. Various preclinical studies linked the lower level of circulating PCSK9 with decreased serum cholesterol levels. In humans, most of the initial trials involving PCSK9 inhibition used monoclonal antibodies like evolocumab and alirocumab directed against PSCK9 that bind this enzyme and cause its degradation.

However, the advent of small interfering RNA (siRNA) opened up a new avenue of genetic manipulation by silencing particular genes [3]. siRNA is a double-stranded RNA molecule that degrades mRNA sequences after transcription, hence preventing mRNA translation, ultimately leading to a decline in the production of the enzymes coded by those genes [4]. For PCSK9, one such drug, inclisiran, is being evaluated in a series of clinical trials-a small interfering RNA that blocks the production of hepatic PCSK9.

Many trials have been undertaken to calculate the safety profile and efficacy of inclisiran in a wide array of populations. The ORION trials are specifically designed to assess treatment outcomes of inclisiran in the broader population [5]. Some of the trials are still underway. Recently, initial results from the primary phase 3 clinical trials have been reported. More extensive trials with more participants with co-morbidities are underway. In this systematic review, we will look at the safety and efficacy outcomes reported in all the clinical trials involving inclisiran to better understand its associated clinical outcomes.

\section{Review}

\section{Literature search}

A broad literature search was done on PubMed, Cochrane, Embase, Scopus, CINAHL, Web of Science, and Clinicaltrials.gov using the terms "Inclisiran," "ALN-PCSsc," and "ALN-PCS." These terms were combined using the Boolean operator "OR.” The search was not limited to any region or any language if the English translation was available. The data cut-off date for this search was June 28, 2020. Relevant articles from recent conferences were also included. 


\section{Cureus}

\section{Eligibility criteria}

Only those studies that met the following criteria were included in the review: (a) inclisiran was the primary intervention drug; (b) original clinical studies with the human population; (c) safety and efficacy outcomes were reported.

\section{Study selection}

For the study, relevant studies were reviewed based on the title and abstract. The potential articles obtained from the first review were then screened again with full text by the same reviewers. Conference abstracts and presentations reporting the interim results of the ongoing inclisiran trials were also included.

\section{Data extraction}

Data extraction was done using pre-specified tables of baseline characteristics, safety, and efficacy. The parameters recorded were author, year, patient population, age, regimen, baseline BMI, baseline LDL cholesterol, and any changes in LDL-cholesterol, PCSK9, and adverse events. The data was recorded as mean, medians, and percentages. The data is demonstrated in Tables 1-3. The PRISMA diagram for the screening of studies is attached (Figure 1).

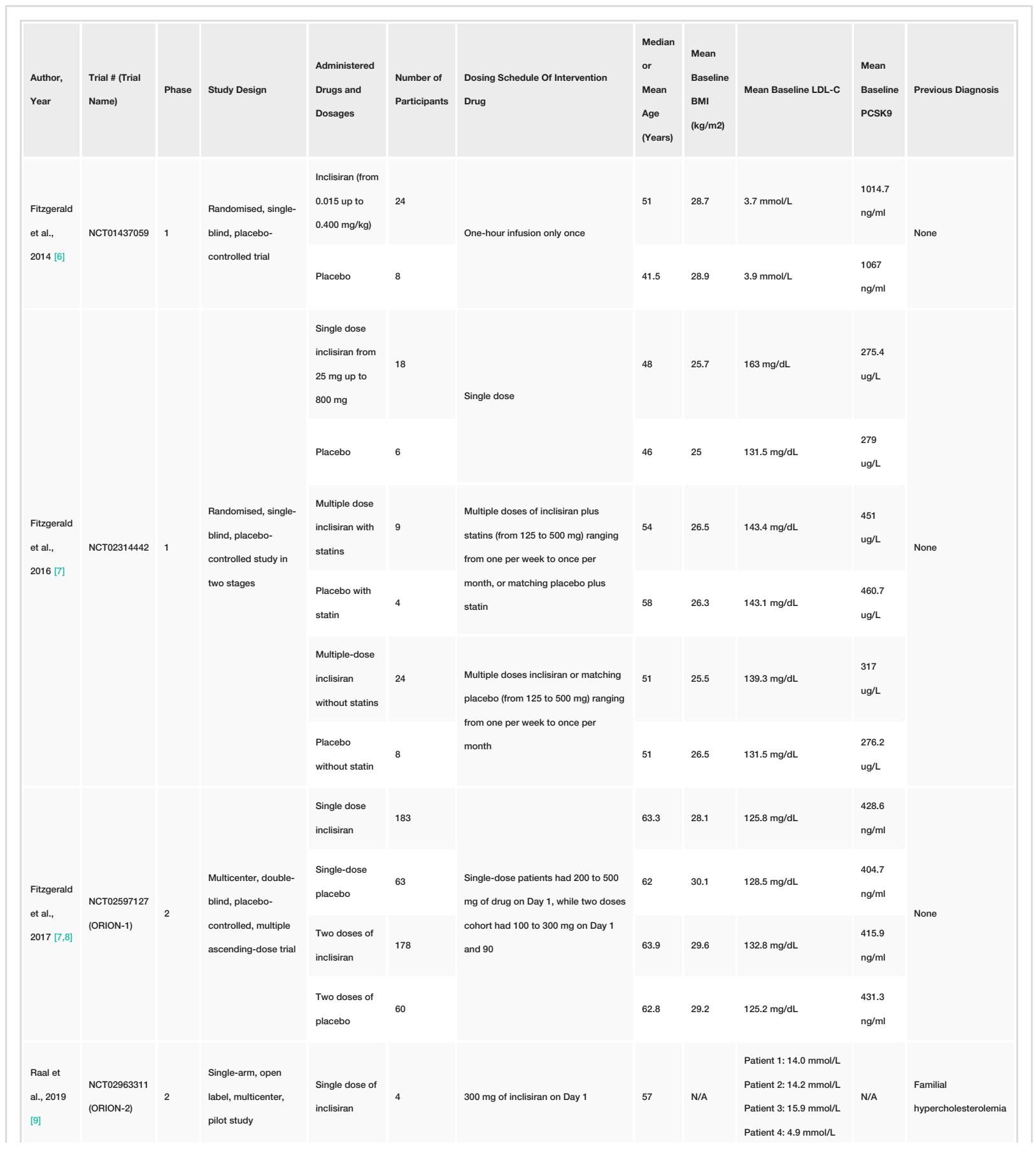




\section{Cureus}

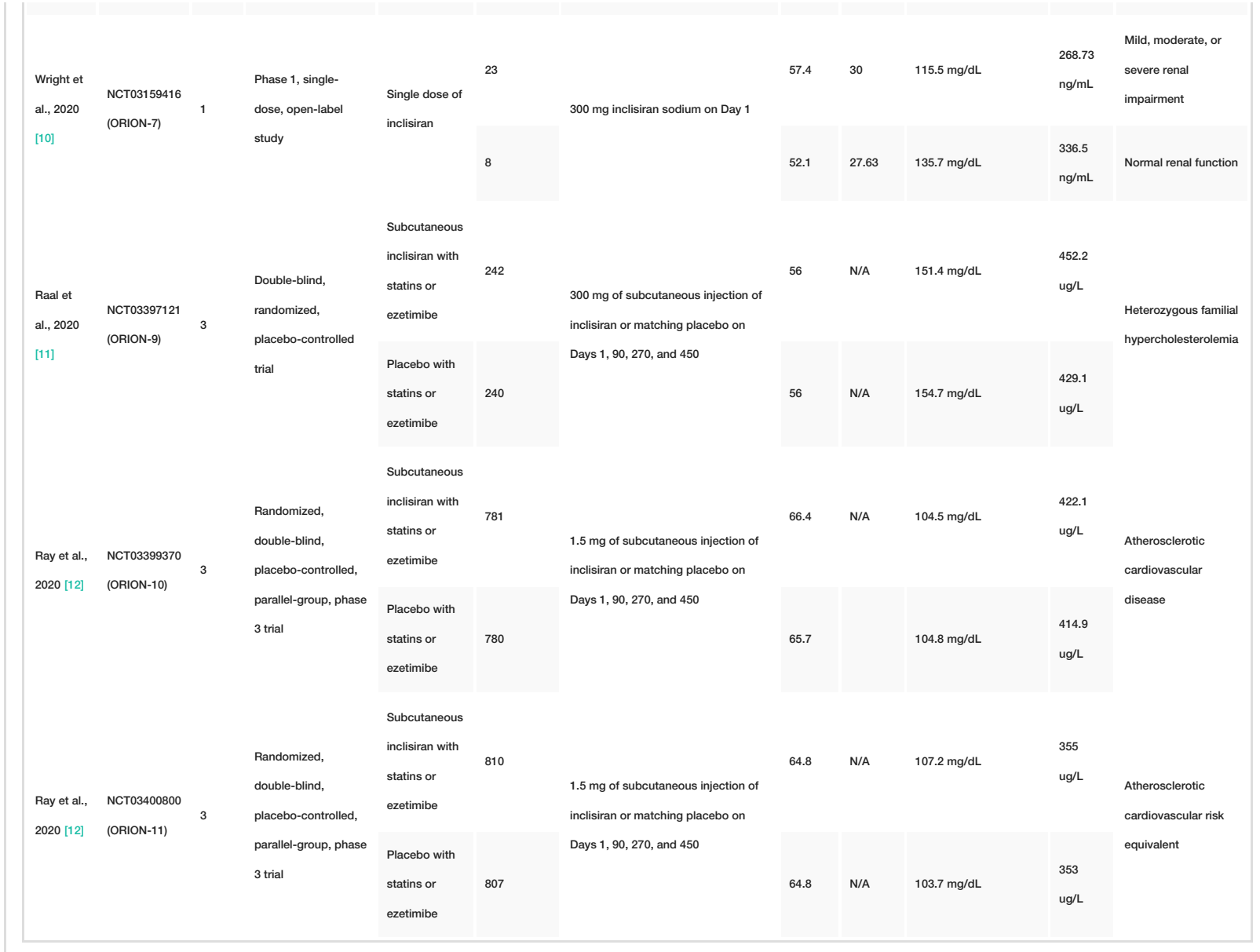

TABLE 1: Baseline characteristics of participants involved in review 


\section{Cureus}

\begin{tabular}{|c|c|c|c|c|}
\hline \multirow{2}{*}{ Author, Year } & \multirow{2}{*}{ Trial name } & \multicolumn{3}{|c|}{ Most common adverse events (number of events) } \\
\hline & & Adverse events & Inclisiran Group & Placebo Group \\
\hline \multirow[t]{4}{*}{ Fitzgerald et al., 2014 [6] } & N/A & Infusion site hematoma & 1 & 1 \\
\hline & & Paresthesia & 3 & \\
\hline & & Rash & 12 & 4 \\
\hline & & Headache & 5 & 2 \\
\hline \multirow[t]{4}{*}{ Fitzgerald et al., 2016 [7] } & N/A & Nasopharyngitis & 7 & N/A \\
\hline & & Diarrhea & 4 & \\
\hline & & Back pain & 5 & \\
\hline & & Headache & 6 & \\
\hline \multirow[t]{2}{*}{ Fitzgerald et al., $2017[7,8]$} & ORION-1 & Injection site reaction & 19 & -- \\
\hline & & Myalgias & 27 & 6 \\
\hline \multirow[t]{2}{*}{ Raal et al., 2019 [9] } & ORION-2 & Paresthesia & 11 & N/A \\
\hline & & Unstable angina & 1 & \\
\hline \multirow[t]{4}{*}{ Wright et al., 2020 [10] } & ORION-7 & Cough & 4 & N/A \\
\hline & & Nasopharyngitis & 1 & \\
\hline & & Headache & 2 & \\
\hline & & Fatigue & 2 & \\
\hline \multirow[t]{5}{*}{ Raal et al., 2020 [11] } & ORION-9 & Nasopharyngitis & 28 & 20 \\
\hline & & Influenza & 13 & 21 \\
\hline & & Upper respiratory tract infections & 16 & 16 \\
\hline & & Back pain & 17 & 10 \\
\hline & & Injection site reaction & 22 & 0 \\
\hline \multirow[t]{4}{*}{ Ray et al., 2020 [12] } & ORION-10 & Death from cardiovascular events & 7 & 5 \\
\hline & & Diabetes mellitus & 120 & 108 \\
\hline & & Bronchitis & 46 & 30 \\
\hline & & Injection site reaction (mild and moderate) & 20 & 7 \\
\hline \multirow[t]{4}{*}{ Ray et al., 2020 [12] } & ORION-11 & Death from cardiovascular events & 9 & 10 \\
\hline & & Injection site reaction & 38 & 4 \\
\hline & & Nasopharyngitis & 91 & 90 \\
\hline & & Diabetes mellitus & 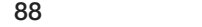 & 94 \\
\hline
\end{tabular}

\section{TABLE 2: Safety of inclisiran}

$\mathrm{N} / \mathrm{A}=$ not available

\begin{tabular}{|c|c|c|c|c|c|}
\hline $\begin{array}{l}\text { Author, } \\
\text { Year }\end{array}$ & $\begin{array}{l}\text { Trial } \\
\text { name }\end{array}$ & $\begin{array}{l}\text { Duration to } \\
\text { response } \\
\text { measurement } \\
\text { (Days) }\end{array}$ & $\begin{array}{l}\text { Administered drugs } \\
\text { and dosages }\end{array}$ & $\begin{array}{l}\text { PCSK9 levels mean } \\
\text { percentage change from } \\
\text { baseline }\end{array}$ & $\begin{array}{l}\text { LDL Cholesterol levels mean } \\
\text { percentage change from } \\
\text { baseline }\end{array}$ \\
\hline & & & Inclisiran (from 0.015 & $-30.8 \%$ at lowest dose & $-14.4 \%$ for lowest dose \\
\hline
\end{tabular}




\section{Cureus}

\begin{tabular}{|c|c|c|c|c|c|c|c|}
\hline \multirow{2}{*}{$\begin{array}{l}\text { Fitzgerald } \\
\text { et al., } \\
2014^{\star}\end{array}$} & \multirow[t]{2}{*}{ N/A } & \multirow[t]{2}{*}{28} & up to $0.400 \mathrm{mg} / \mathrm{kg}$ ) & \multicolumn{2}{|c|}{$-58.6 \%$ at highest dose } & \multicolumn{2}{|c|}{$-36.1 \%$ for highest dose } \\
\hline & & & Placebo & \multicolumn{2}{|l|}{$-8.7 \%$} & \multicolumn{2}{|l|}{$-24 \%$} \\
\hline \multirow{8}{*}{$\begin{array}{l}\text { Fitzgerald } \\
\text { et al., 2016* } \\
\text { [7] }\end{array}$} & \multirow{8}{*}{ N/A } & \multirow{8}{*}{84} & Single-dose inclisiran & \multicolumn{2}{|c|}{$-46.6 \%$ at lowest dose } & \multicolumn{2}{|c|}{$-21.5 \%$ at lowest dose } \\
\hline & & & $\mathrm{mg}$ & \multicolumn{2}{|c|}{$-73.1 \%$ at highest dose } & \multicolumn{2}{|c|}{$-43.4 \%$ at highest dose } \\
\hline & & & Placebo & \multicolumn{2}{|l|}{$-0.6 \%$} & \multicolumn{2}{|l|}{$-10.9 \%$} \\
\hline & & & Multiple-dose inclisiran & \multicolumn{2}{|c|}{$-79.9 \%$ at lowest dose } & \multicolumn{2}{|c|}{$-45.1 \%$ at lowest dose } \\
\hline & & & with statıns & \multicolumn{2}{|c|}{$-83.8 \%$ at highest dose } & \multicolumn{2}{|c|}{$-53.2 \%$ at highest dose } \\
\hline & & & Multiple-dose inclisiran & \multicolumn{2}{|c|}{$-75.7 \%$ at lowest dose } & \multicolumn{2}{|c|}{$-52.2 \%$ at lowest dose } \\
\hline & & & without statins & \multicolumn{2}{|c|}{$-81.5 \%$ at highest dose } & \multicolumn{2}{|c|}{$-51.7 \%$ at highest dose } \\
\hline & & & Placebo & \multicolumn{2}{|l|}{$+16.9 \%$} & \multicolumn{2}{|l|}{$-14.2 \%$} \\
\hline & & & & \multicolumn{2}{|c|}{$-47.9 \%$ at lowest dose } & \multicolumn{2}{|c|}{$-27.9 \%$ at lowest dose } \\
\hline & & & & \multicolumn{2}{|c|}{$-59.3 \%$ at highest dose } & \multicolumn{2}{|c|}{$-41.9 \%$ at highest dose } \\
\hline $\begin{array}{l}\text { Fitzgerald } \\
\text { et } 2017^{\star}\end{array}$ & ORION- & & Single-dose placebo & $+2.2 \%$ & & $+2.1 \%$ & \\
\hline & & & Two doses of & $-53.2 \%$ at low & dose & $-35.5 \%$ at lo & lose \\
\hline & & & inclisiran & $-69.1 \%$ at hig & t dose & $-52.6 \%$ at hic & dose \\
\hline & & & Two doses of placebo & $-1.2 \%$ & & $+1.8 \%$ & \\
\hline & & & & Patient A: -48 & & Patient $A:+1$ & \\
\hline Raal et al., & ORION- & & & Patient B: -51 & & Patient B: -1 & \\
\hline $2019^{\star \star}[9]$ & 2 & & & Patient C: -51 & & Patient C: -3 & \\
\hline & & & & Patient D: -83 & & Patient D: -1 & \\
\hline & & & & Mild RI & $-74.2 \%$ & Mild RI & $-35.1 \%$ \\
\hline $\begin{array}{l}\text { Wright et } \\
\text { al., } 2020\end{array}$ & ORION- & & & Moderate RI & $-79.8 \%$ & Moderate RI & $-53.1 \%$ \\
\hline & $r$ & & & Severe RI & $-67.9 \%$ & Severe RI & $-49.2 \%$ \\
\hline & & & & Normal RF & $-68.1 \%$ & Normal RF & $-57.6 \%$ \\
\hline $\begin{array}{l}\text { Raal et al., } \\
2020[11]\end{array}$ & $\begin{array}{l}\text { ORION- } \\
9\end{array}$ & 510 & $\begin{array}{l}\text { Subcutaneous } \\
\text { inclisiran with statins } \\
\text { or ezetimibe }\end{array}$ & $-60.7 \%$ & & $-39.7 \%$ & \\
\hline & & & $\begin{array}{l}\text { Placebo with statins or } \\
\text { ezetimibe }\end{array}$ & $+17.7 \%$ & & $+8.2 \%$ & \\
\hline Ray et al., & ORI & 510 & $\begin{array}{l}\text { Subcutaneous } \\
\text { inclisiran with statins } \\
\text { or ezetimibe }\end{array}$ & $-69.8 \%$ & & $-51.3 \%$ & \\
\hline & & & $\begin{array}{l}\text { Placebo with statins or } \\
\text { ezetimibe }\end{array}$ & $+13.5 \%$ & & $+1.0 \%$ & \\
\hline $\begin{array}{l}\text { Ray et al., } \\
2020 \text { [12] }\end{array}$ & $\begin{array}{l}\text { ORION- } \\
11\end{array}$ & 510 & $\begin{array}{l}\text { Subcutaneous } \\
\text { inclisiran with statins } \\
\text { or ezetimibe }\end{array}$ & $-63.6 \%$ & & $-45.8 \%$ & \\
\hline & & & $\begin{array}{l}\text { Placebo with statins or } \\
\text { ezetimibe }\end{array}$ & $+15.6 \%$ & & $+4.0 \%$ & \\
\hline
\end{tabular}

\section{TABLE 3: Efficacy of inclisiran}

RI: renal impairment

*In these multiple dosing groups studies, the efficacy is written for only the highest and the lowest doses. 


\section{Cureus}

${ }^{\star \star}$ This is a single-arm trial.
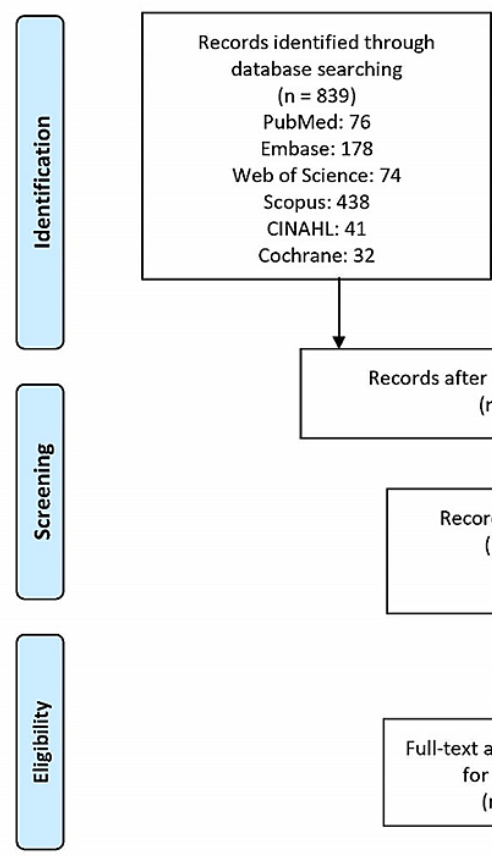

Records after duplicates removed ( $n=560$ )
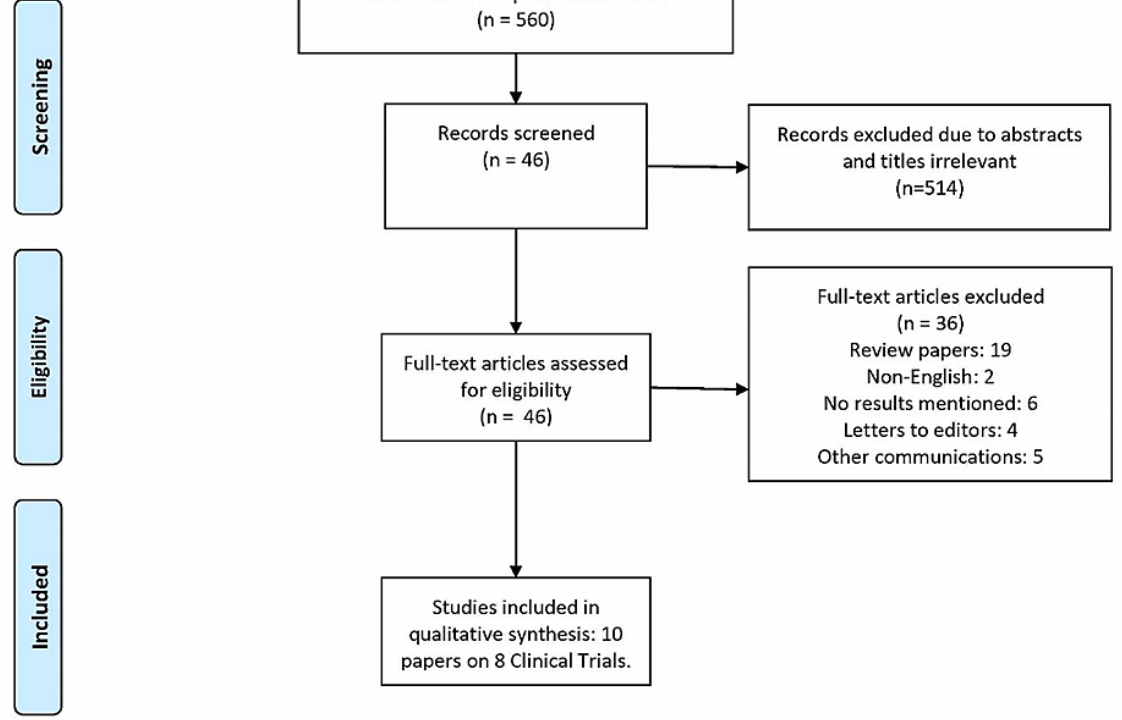

Fig. 1 : PRISMA Flowchart of Selection and Screening of Studies

FIGURE 1: PRISMA diagram for screening of clinical studies.

\section{Results}

The literature search yielded a total of 839 articles. After excluding 291 duplicates, 560 articles were screened based on titles and abstracts. After this first screening, 46 studies were found potentially helpful for our review after excluding 514 items. Full texts of these 46 articles were reviewed, and a further 36 articles were excluded due to one of the following reasons: review papers $(n=19)$, outcomes not measured $(n=6)$, English translation not available $(n=2)$, letters to editors, and other communications $(n=9)$. This study selection and screening process are depicted in Figure 1. Ten articles based on eight clinical trials were included in the final review.

\section{Pre-ORION Trials}

Before the ORION trials, two clinical studies were done, and the results were reported in 2014 and 2016, respectively [6,7]. Both were phase 1 randomised single-blinded placebo-controlled trials. In the first trial $(n=32$, inclisiran group $=24$, placebo arm $=8)$, patients in inclisiran group were given inclisiran single infusion $(0.015 \mathrm{mg} / \mathrm{kg}$ to $0.4 \mathrm{mg} / \mathrm{kg})$. This led to a decrease ranging from $30.8 \%$ to $58.5 \%$ in levels of PCSK9 from baseline compared to only an $8.7 \%$ decrease in the placebo group. Similarly, the decrease in LDL cholesterol levels ranged from $14.4 \%$ to $36.1 \%$ from baseline compared to a $24 \%$ decrease in the placebo group.

In the latter trial $(n=69$, inclisiran=51, placebo=18), the inclisiran group patients were given either a single dose of $25 \mathrm{mg}$ to $800 \mathrm{mg}$ inclisiran or multiple doses 125 to $500 \mathrm{mg}$ inclisiran once a week to once a month with or without statins. For the single-dose cohort, the decrease in PCSK9 levels ranged from $46.6 \%$ to $73.1 \%$. In the multiple doses' cohorts, this decrease ranged from $79.9 \%$ to $83.8 \%$ with statins, and $75.1 \%$ to 
81.5\% without statins, compared to an increase in PCSK9 levels by $16.9 \%$ in the placebo arm. In comparison, the range of decrease in LDL cholesterol levels in these three cohorts were $21.5 \%$ to $43.1 \%, 45.1 \%$ to $53.2 \%$, and $51.7 \%$ to $52.2 \%$, respectively.

These trials demonstrated the safety of inclisiran with mild to moderate adverse events, which commonly occurred in both treatment and placebo groups. The most common side effects included injection site reactions, musculoskeletal pain, nasopharyngitis, headache, back pain, and diarrhea. No clinically significant and trending changes were seen on comprehensive metabolic panels, hepatic function tests, cardiac enzymes and inflammatory markers on initiation at six months follow-up. No treatment discontinuations were reported [6,7].

ORION-1

ORION-1 was the first trial of the ORION series to establish the safety and efficacy of inclisiran as a cholesterol-lowering drug. It was a multicenter, double-blind, placebo-controlled trial. The first dose of drug/placebo was given on day 1 , and the second dose was given on day 90 . In the single-dose group, the percent decrease in PCSK9 levels from baseline ranged from $47.9 \%$ to $59.3 \%$, while it increased by $2.2 \%$ for the placebo group. For the two doses group, the PCSK9 levels decreased by $75.7 \%$ at the lowest dose and $81.5 \%$ at the highest dose. In contrast, PCSK9 levels increased by $16.9 \%$ for the placebo group. Similarly, the decrease in LDL cholesterol levels ranged from $27.9 \%$ to $41.9 \%$ and $35.5 \%$ to $52.6 \%$ in the single and two doses groups.

ORION-1 trial showed the first treatment discontinuations for one patient in the placebo group developing herpes zoster and another developing nasopharyngitis/influenza in the treatment arm. The equivalent occurrence of mild adverse effects, including dizziness, myalgia, headache, fatigue, back pain, hypertension, and diarrhea in individuals getting Inclisiran and placebo, were seen. Injection-site reactions commonly occurred in the inclisiran arm. Transient liver enzyme elevations were seen in both groups with no evidence of hepatocellular injury to warrant drug discontinuation at any point in the trial [8].

\section{ORION-2}

The interim results of the ORION-2 trial were presented at the Conference of European Atherosclerotic society in 2019. This trial was intended to profile inclisiran in patients with homozygous familial hypercholesterolemia $(\mathrm{HoFH})$. In the interim results, the responses in four patients were reported. The baseline LDL-C levels in this cohort were 14, 14.2, 15.9, and $4.9 \mathrm{mmol} / \mathrm{L}$. On day 90, the LDL-C increased by $14.3 \%$ in the first patient but was decreased by $11.7 \%, 33.1 \%$, and $17.5 \%$, respectively, in the other patients. But PCSK9 levels decreased in all four patients by 48.7\%, 51.6\%, 51.7\%, and 83.6\%, respectively. No safety results have been reported [9].

ORION-7

ORION-7 was a phase 1 open-label study designed to test the efficacy of inclisiran in patients with renal insufficiency compared to individuals with normal renal function. All patients were given a single dose of $300 \mathrm{mg}$ Inclisiran. PCSK9 levels decreased by $74.2 \%, 79.8 \%$, and $67.9 \%$ in mild, moderate, and severe renal insufficiency, while it decreased by $68.1 \%$ in patients with normal renal insufficiency. Similarly, the LDL-C decreased by $35.1 \%, 53.1 \%$, and $49.2 \%$ in patients with mild, moderate, and severe renal insufficiency and decreased by $57.6 \%$ in the normal renal function group [10].

Inclisiran was also well-tolerated in groups with normal kidney function and those with mild, moderate, and severe renal injury in ORION-7. No requirement for dose adjustment in patients with renal impairment was warranted for the same or more reduction in LDL levels based on the data reported [10].

ORION-9

ORION-9 was a phase 3, double-blind, placebo-controlled trial to assess the efficacy of inclisiran in patients with heterozygous familial hypercholesterolemia. The treatment group $(\mathrm{n}=242)$ was administered $300 \mathrm{mg}$ of inclisiran on Days 1, 90, 270, and 450, while the control group $(n=240)$ was given a placebo. The mean PCSK9 levels decreased by $60.7 \%$ in the inclisiran group on 510 th day follow up, but it was increased by $17.7 \%$ in the placebo group. Similarly, the mean LDL cholesterol decreased by $39.7 \%$ in the inclisiran group but was increased by $8.2 \%$ in the control group [11].

The commonly reported adverse events in the inclisiran arm were nasopharyngitis, back pain, and upper respiratory tract infections. However, there was no statistically significant difference in these events' risk ratios in the treatment and control groups. The only significant side effect in the treatment group was injection site reaction $(\mathrm{n}=22)$, and none of those reactions was graded as severe or persistent [11].

ORION-10 and -11 
ORION-10 ( $\mathrm{n}=1561)$ and ORION-11 $(\mathrm{n}=1617)$ were two similarly designed placebo-controlled trials to assess the efficacy of inclisiran in a larger population. The patients included in these two trials had atherosclerotic cardiovascular disease or were at high risk of it. The results of both these phase 3 trials are published in 2020. The inclisiran group patients were given $1.5 \mathrm{ml}$ of subcutaneous injection of inclisiran on Days 1,90 , 270 , and 450 . The mean PCSK9 levels at day 510 were decreased by $69.8 \%$ and $63.6 \%$ in treatment groups of ORION-10 and ORION-11, respectively. But it increased by $13.5 \%$ and $15.6 \%$ in the control group of both these trials, respectively. Moreover, the LDL cholesterol also decreased by $51.3 \%$ and $45.8 \%$ in the inclisiran group of ORION-10 and -11, respectively, but it increased by $1 \%$ and $4 \%$ in control groups of these trials.

As with ORION-9, the only statistically significant adverse event occurring in the treatment arm were injection site reactions in both trials. However, all of them were graded as mild or moderate [12].

\section{Discussion}

The relationship between low levels of LDL-C and improved cardiovascular outcomes is well established. Higher LDL levels were associated with a higher ASCVD score with a consequent more significant risk of forthcoming adverse cardiovascular events [13]. Mortality rates were improved even in younger populations with higher LDL levels. Lipid-lowering drugs were studied to reduce mortality, and statins were and are one of the foremost lipid-lowering drugs [14]. They are also used for their anti-inflammatory effect in patients with recent stroke and myocardial infarction to stabilise atherosclerotic plaque [15]. The reduction in adverse events and mortality rate was even seen in patients with baseline low LDL levels treated with LDL lowering pharmacological therapy.

The mechanism of action of inclisiran is based on PCSK9 inhibition, which dictates the LDL levels circulating in serum by enzyme-mediated degradation of LDL receptors [16]. This mechanism's idea of pharmacological antagonism, giving birth to inclisiran and PSCK9 antibodies, originated from studying the gain of function mutation for the PCSK9 gene in inherently predisposed individuals. They were seen usually clustered in families. The gain of function of the PCSK gene, which encodes NARC-1 (neural apoptosis regulated convertase) contributing to cholesterol homeostasis, is studied as the basis for familial hypercholesterolemia. This entity is a known atherosclerotic risk factor predisposing individuals to adverse cardiovascular events and premature CAD [17-19].

Similarly, the opposite phenomenon with a mutation of this gene with a diminution of function was observed in specific population groups with lower LDL levels and better cardiovascular outcomes and reduced mortality [3, 20-25]. A reduced incidence of myocardial infarction and even aortic valve stenosis was seen in these patients with lower PCSK9 expression [26]. A study was carried out in Japan to study the genetic mutations in patients, which reiterated the same results [27]. Another large cohort study is on its way to establish the association of PCSK9 mutations with LDL levels and consequent cardiac event(s) [28].

The efficacy of inclisiran in reducing LDL levels has been evident in all the clinical trials included in our study. The initial phase 1 studies were randomised and single-blind studies; reduction of LDL-C was seen in both single-dose and multiple-dose studies [6,7]. Subsequent trials were multi-centric, double-blind, placebo-controlled studies. They demonstrated the same results, albeit with more statistical significance due to larger cohorts including a healthier population and the patients with mild, moderate to severe renal insufficiency, known atherosclerotic disease, or at risk of adverse cardiovascular events and in patients with hypercholesterolemia. There was one, possibly, aberrant reporting of increased LDL in one patient in ORION-2 [9], but the PCSK9 levels were lowered in all participants. That patient's LDL levels were previously resistant to anti-PCSK9 antibodies. It may suggest a guideline for avoiding the use of inclisiran and similar drugs in the future in the specific patient cohort. Inclisiran has the edge over prior lipid-lowering medications due to a large margin of efficacy and convenient dosing intervals $[8,11,12]$.

The majority of the adverse effects in all trials included in our study were classified as mild to moderate and were manageable with no long-term side effects (Table 2). Safety and efficacy in initial tests were questioned due to the smaller sample size. A large number of the patients from the sample size in ORION=1 belonged to the Caucasian group [8]. The efficacy could not be well demonstrated for the multi-dose phases as subsequent doses of inclisiran if administered within the first dose's maximal pharmacodynamics activity, which could lead to apparently moderate incremental effects of increasing amounts on the levels of PCSK9 and LDL cholesterol.

The number of participants was then increased in randomised subsequent trials to assess safety profiles and pharmacodynamics of inclisiran better. The skin site reactions, including rash, were reported as mild and transient and occurred with similar frequency in both groups (Table 2). A possible underlying ascertainment bias was reported in the background due to over-reporting of this side effect from one particular clinical site in pre-ORION trials [6,7]. No long-term skin changes were observed. Considering the premedication if administered and proper skin reaction classification using a predefined criterion with appropriate follow-up can help reduce this adverse effect.

Two fatalities were reported later in the ORION-1 trial. A patient assigned to the single-dose 500-mg 
inclisiran group with a long-standing peripheral vascular disease and recurrent anginas who underwent a cardiac arrest died on Day 104. Another man in the two-dose 200-mg inclisiran group died on day 198 in trial with a surgical repair of thoracic aortic aneurysm and subsequent complication by fistula and sepsis after participation in the trial. The attribution of any of these fatal outcomes to inclisiran has been difficult to explain due to multiple comorbidities and unforeseeable health calamities, which had no basis for a possible drug-related reaction. Platelet levels and basic metabolic panels were stable, and no other abnormalities were documented [8].

Elevated gamma-glutamyl transferase (GGT) was reported in a patient with concomitant statin therapy, which improved on discontinuation and recurred on initiation, classifying this elevation as most likely related to statin therapy. Initial studies had a meagre number of patients already taking statins, and intake of additional lipid-lowering drugs like ezetimibe or others was not screened [6,7]. Subsequent inclusion of patients with known atherosclerotic disease or at high risk for it with randomisation ensured an appropriate representation of patients already receiving statins, and no possible drug-drug interactions were reported. ORION-9 trial showed low-titer antidrug antibodies consistent with assay-testing characteristics and not considered to be due to treatment with inclisiran [9]. The presence of these antibodies was transient and not associated with changes in any pharmacologic or clinical measurements.

The long-term results of phase 3 LDL-C lowering studies (ORION trials 9, 10,11) and results of ongoing clinical trials like ORION-4 will provide us with more data to comment on the renal and cardiovascular safety of inclisiran. The efficacy of injecting inclisiran twice is reported to be greater than one-time dosing in earlier clinical studies. The data from future trials will help us take a proper prophylactic and preventive approach in these patients while prescribing inclisiran or recruiting patients in subsequent trials. Previously used lipid-lowering drugs have an established efficacy but an undesirable side effect profile, including elevated liver enzymes, drug interactions, and rhabdomyolysis [28]. Paradoxically, some studies showed that the PCSK9 gene levels were upregulated in patients taking statins as lipid-lowering agents, but more studies are needed to prove this causation [29]. The frequency of administration of inclisiran is convenient (twice yearly compared to every four to six weeks for the monoclonal antibodies). The final pricing for inclisiran has not been commented upon but is fairly priced compared to the monoclonal antibodies. Inclisiran can emerge as a superior substitute for lowering both serum LDL levels and subsequent risks of adverse cardiovascular events.

\section{Conclusions}

In light of the recent randomized placebo-controlled trials, inclisiran has proven efficacious in a wide variety of patient populations. Inclisiran has shown a promising safety profile. Severe adverse events and fatalities noted in trials have not been related to inclisiran. The results of the ongoing clinical trials will help us to corroborate these findings further. They would open vistas for the potential application of inclisiran in suitable and responsive patient populations.

\section{Additional Information \\ Disclosures}

Conflicts of interest: In compliance with the ICMJE uniform disclosure form, all authors declare the following: Payment/services info: All authors have declared that no financial support was received from any organization for the submitted work. Financial relationships: All authors have declared that they have no financial relationships at present or within the previous three years with any organizations that might have an interest in the submitted work. Other relationships: All authors have declared that there are no other relationships or activities that could appear to have influenced the submitted work.

\section{References}

1. Page MM, Watts GF: PCSK9 inhibitors - mechanisms of action. Aust Prescr. 2016, 39:164-7. 10.18773/austprescr.2016.060

2. Rashid S, Curtis DE, Garuti R, et al.: Decreased plasma cholesterol and hypersensitivity to statins in mice lacking Pcsk9. Proc Natl Acad Sci U S A. 2005, 102:5374-9. 10.1073/pnas.0501652102

3. Elbashir SM, Harborth J, Lendeckel W, Yalcin A, Weber K, Tuschl T: Duplexes of 21-nucleotide RNAs mediate RNA interference in cultured mammalian cells. Nature. 2001, 411:494-8. 10.1038/35078107

4. Agrawal N, Dasaradhi PV, Mohmmed A, Malhotra P, Bhatnagar RK, Mukherjee SK: RNA interference: biology, mechanism, and applications. Microbiol Mol Biol Rev. 2003, 67:657-85. 10.1128/MMBR.67.4.657685.2003

5. Stoekenbroek RM, Kallend D, Wijngaard PL, Kastelein JJ: Inclisiran for the treatment of cardiovascular disease: the ORION clinical development program. Future Cardiol. 2018, 14:433-42. 10.2217/fca-2018-0067

6. Fitzgerald K, Frank-Kamenetsky M, Shulga-Morskaya S, et al.: Effect of an RNA interference drug on the synthesis of proprotein convertase subtilisin/kexin type 9 (PCSK9) and the concentration of serum LDL cholesterol in healthy volunteers: a randomised, single-blind, placebo-controlled, phase 1 trial. Lancet. 2014, 383:60-8. 10.1016/S0140-6736(13)61914-5

7. Fitzgerald K, White S, Borodovsky A, et al.: A highly durable RNAi therapeutic inhibitor of PCSK9 . N Engl J Med. 2017, 376:41-51. 10.1056/NEJMoa1609243

8. Ray KK, Landmesser U, Leiter LA, et al.: Inclisiran in patients at high cardiovascular risk with elevated LDL 
cholesterol. N Engl I Med. 2017, 376:1430-40. 10.1056/NEJMoa1615758

9. Raal F, Lepor N, Kallend D, Stoekenbroek R, Wijngaard P, Hovingh GK:: Inclisiran durably lowers LDL-C and PCSK9 expression in subjects with homozygous familial hypercholesterolemia: the Orion-2 pilot study. Atherosclerosis. 2019, 287:7-7. 10.1016/j.atherosclerosis.2019.06.018

10. Wright RS, Collins MG, Stoekenbroek RM, et al.: Effects of renal impairment on the pharmacokinetics, efficacy, and safety of inclisiran: an analysis of the ORION-7 and ORION-1 Studies. Mayo Clin Proc. 2020, 95:77-89. 10.1016/j.mayocp.2019.08.021

11. Raal FJ, Kallend D, Ray KK, et al.: Inclisiran for the treatment of heterozygous familial hypercholesterolemia. N Engl J Med. 2020, 382:1520-30. 10.1056/NEJMoa1913805

12. Ray KK, Wright RS, Kallend D, et al.: Two phase 3 trials of inclisiran in patients with elevated LDL cholesterol. N Engl J Med. 2020, 382:1507-19. 10.1056/NEJMoa1912387

13. Hsia J, MacFadyen JG, Monyak J, Ridker PM: Cardiovascular event reduction and adverse events among subjects attaining low-density lipoprotein cholesterol \&lt; $50 \mathrm{mg} / \mathrm{dl}$ with rosuvastatin. The JUPITER trial (Justification for the Use of Statins in Prevention: an Intervention Trial Evaluating Rosuvastatin). J Am Coll Cardiol. 2011, 57:1666-75. 10.1016/j.jacc.2010.09.082

14. Law MR, Wald NJ, Rudnicka AR: Quantifying effect of statins on low density lipoprotein cholesterol, ischaemic heart disease, and stroke: systematic review and meta-analysis. BMJ. 2003, 326:1423. 10.1136/bmj.326.7404.1423

15. Singh P, Emami H, Subramanian S, et al.: Coronary plaque morphology and the anti-inflammatory impact of atorvastatin: a multicenter 18F-fluorodeoxyglucose positron emission tomographic/computed tomographic study. Circ Cardiovasc Imaging. 2016, 9:e004195. 10.1161/CIRCIMAGING.115.004195

16. Horton JD, Cohen JC, Hobbs HH: Molecular biology of PCSK9: its role in LDL metabolism . Trends Biochem Sci. 2007, 32:71-7. 10.1016/j.tibs.2006.12.008

17. Abifadel M, Varret M, Rabès JP, et al.: Mutations in PCSK9 cause autosomal dominant hypercholesterolemia. Nat Genet. 2003, 34:154-6. 10.1038/ng1161

18. Watts GF, Gidding S, Wierzbicki AS, et al.: Integrated guidance on the care of familial hypercholesterolaemia from the International FH Foundation. Int J Cardiol. 2014, 171:309-25. 10.1016/.ijcard.2013.11.025

19. Nordestgaard BG, Chapman MJ, Humphries SE, et al.: Familial hypercholesterolaemia is underdiagnosed and undertreated in the general population: guidance for clinicians to prevent coronary heart disease: consensus statement of the European Atherosclerosis Society. Eur Heart J. 2013, 34:3478-90a. 10.1093/eurhearti/eht273

20. Cohen JC, Boerwinkle E, Mosley TH Jr, Hobbs HH: Sequence variations in PCSK9, low LDL, and protection against coronary heart disease. N Engl J Med. 2006, 354:1264-72. 10.1056/NEJMoa054013

21. Cohen J, Pertsemlidis A, Kotowski IK, Graham R, Garcia CK, Hobbs HH: Low LDL cholesterol in individuals of African descent resulting from frequent nonsense mutations in PCSK9. Nat Genet. 2005, 37:161-5. $10.1038 / \mathrm{ng} 1509$

22. Kotowski IK, Pertsemlidis A, Luke A, Cooper RS, Vega GL, Cohen JC, Hobbs HH: A spectrum of PCSK9 alleles contributes to plasma levels of low-density lipoprotein cholesterol. Am J Hum Genet. 2006, 78:410-22. $10.1086 / 500615$

23. Zhao Z, Tuakli-Wosornu Y, Lagace TA, et al.: Molecular characterization of loss-of-function mutations in PCSK9 and identification of a compound heterozygote. Am J Hum Genet. 2006, 79:514-23. 10.1086/507488

24. Hooper AJ, Marais AD, Tanyanyiwa DM, Burnett JR: The C679X mutation in PCSK9 is present and lowers blood cholesterol in a Southern African population. Atherosclerosis. 2007, 193:445-8. 10.1016/j.atherosclerosis.2006.08.039

25. Benn M, Nordestgaard BG, Grande P, Schnohr P, Tybjaerg-Hansen A: PCSK9 R46L, low-density lipoprotein cholesterol levels, and risk of ischemic heart disease: 3 independent studies and meta-analyses. J Am Coll Cardiol. 2010, 55:2833-42. 10.1016/j.jacc.2010.02.044

26. Langsted A, Nordestgaard BG, Benn M, Tybjærg-Hansen A, Kamstrup PR: PCSK9 R46L loss-of-function mutation reduces lipoprotein(a), LDL cholesterol, and risk of aortic valve stenosis. J Clin Endocrinol Metab. 2016, 101:3281-7. 10.1210/jc.2016-1206

27. Mabuchi H, Nohara A, Noguchi T, et al.: Molecular genetic epidemiology of homozygous familial hypercholesterolemia in the Hokuriku district of Japan. Atherosclerosis. 2011, 214:404-7. 10.1016/j.atherosclerosis.2010.11.005

28. Nomura A, Tada H, Okada H, Nohara A, Ishikawa H, Yoshimura K, Kawashiri MA: Impact of genetic testing on low-density lipoprotein cholesterol in patients with familial hypercholesterolemia (GenTLe-FH): a randomised waiting list controlled open-label study protocol. BMJ Open. 2018, 8:e023636. 10.1136/bmjopen-2018-023636

29. Dubuc G, Chamberland A, Wassef H, Davignon J, Seidah NG, Bernier L, Prat A: Statins upregulate PCSK9, the gene encoding the proprotein convertase neural apoptosis-regulated convertase-1 implicated in familial hypercholesterolemia. 2004. 10.1161/01.ATV.0000134621.14315.43 